\title{
Undetectable TSH could be a TSH variant; case of a young asymptomatic Nepalese male
}

\author{
Santosh Pradhan ${ }^{1}$, Vivek Pant ${ }^{1}$, Keyoor Gautam², Devish Pyakurel ${ }^{2}$, Abha Shrestha ${ }^{2}$ \\ ${ }^{1}$ Department of Clinical Biochemistry, Samyak Diagnostic; Jawalakhel, Lalitpur \\ ${ }^{2}$ Department of Pathology, Samyak Diagnostic; Jawalakhel, Lalitpur, Nepal
}

\begin{abstract}
Thyroid function tests are frequently ordered test. Infrequently, the automated thyroid hormone assay system is subjected to interference which may yield false results, thus leading to inappropriate diagnosis and management. We report an unusual case of clinically misdiagnosed subclinical hyperthyroidism with undetectable TSH due to negative interference in particular TSH assay platforms in a young Nepalese male.
\end{abstract}

Key Words: Thyroid stimulating hormone (TSH); TSH variant; Immunoassay;

\section{Introduction}

Thyroid function test is offered as a routine investigation by most of the clinical laboratories. Clinical and biochemical phenotype correlation is required for the correct interpretation of thyroid hormone test result. Measurement of thyroid hormones in serum by immunoassay method is preferred and has also been advocated since many years. ${ }^{1}$ Susceptibility of automated thyroid hormone immunoassays to interference leads to false result, with the potential for inappropriate diagnosis and management. There are many cases, where discordant thyroid stimulating hormone (TSH) measurement in euthyroid subjects is reported. The common causes of interference in thyroid hormone measurement are intake of biotin containing multivitamins by patient and presence of heterophile antibodies, rheumatoid factor or paraprotein in patient's serum. ${ }^{2,3,4}$ It should be noted that high affinity of the noncovalent biotinstreptavidin interaction has been extensively used in competitive immunoassays. Thus, additional immunoassay interference that should be taken into consideration in discordant thyroid hormone measurement is antistreptavidin antibodies,

Corresponding Author

Vivek Pant, MD Biochemistry, Department of Clinical Biochemistry, Samyak Diagnostic; Jawalakhel , Lalitpur, Nepal P.O.Box: 11708 . Email: drvpant@gmail.com anti-ruthenium antibodies and thyroid hormone autoantibodies. ${ }^{5}$ Thyroid hormone immunoassay is based on antibody targeted against two different region of TSH dimer. Whenever the immunogenicity of patients TSH is altered due to change in peptide sequence of TSH, then some immunoassays cannot detect this TSH although the biological function of TSH is retained. This normal TSH variant leads to misdiagnosis of subclinical hyperthyroidism in the absence of clinical symptoms. Here we present a case of 25 years male, who is a medical student and was misdiagnosed with subclinical hyperthyroidism possibly due to TSH variant.

\section{Case Report}

A 25 years old male ordered his thyroid function test (TFT) as a routine checkup along with other tests at one of the private laboratory in Kathmandu. He had no past illness and was completely normal while performing test. He had normal serum free triiodothyronine (T3) and free thyroxine (T4) level but TSH 3rd generation was less than 0.01. He repeated his test after two weeks at same laboratory and the TFT findings were same. He went for endocrinology consultation where he was diagnosed with subclinical hyperthyroidism. He was advised to perform additional test like anti thyroid peroxidase antibody (TPO antibody) and antithyroglobulin. He was also advised to start treatment. He crosschecked 
Undetectable TSH could be a TSH variant; case of...

Jour of Diab and Endo Assoc of Nepal 2019; 3 (1): (34-37)

his TFT few days later before starting treatment at next private laboratory in Kathmandu. This time his serum fT3, fT4 and TSH were in normal range. He came to our laboratory for counseling. He had no viral illness and neck pain during the past 3 months. He was not taking multivitamins or levothyroxine and had no major illness. At this time his reports for anti TPO and antithyroglobulin were available which were negative. We repeated the thyroid function test at our laboratory and again the serum TSH level was less than 0.01. We contacted the laboratories where he had done his tests. In the first laboratory where TSH was undetected, they were using Siemens Advia Centaur XP immunoassay. The second laboratory where the TFT test was normal, were using Roche immunoassay system. At our laboratory we are using both Siemens and Abott Architect immunoassay. We discussed with the patient and repeated the test in Abbott TSH immunoassay system, where the results were normal. The patient was then explained about the TSH variant and was reassured that he does not need treatment for this condition.

\section{Discussion}

TSH is routinely measured in the clinical laboratory to diagnose and monitor thyroid disorders. Thirdgeneration serum TSH assays have the highest sensitivity for assessing thyroid disorders. ${ }^{6}$

Immunoassay system is currently the method of choice in clinical laboratories for the measurement of thyroid hormones. However, immunoassays are vulnerable to different types of interference that can result in discordant result of thyroid function test. Common interferences are due to presence of macro-TSH, biotin, antistreptavidin antibodies, antiruthenium antibodies, and heterophilic antibodies. The manufacturers of immunoassay try to limit their impact of interference by adding blockers in reagent or by warning users via information provided in kit inserts. Ideally, discrepancies with biochemical parameters of TFT and clinical findings provide clue to the interference. Clinical history, regarding intake of multivitamins or other drugs, history of blood transfusion, autoimmune diseases or contact with animals or any discordant lab result in the past, should be taken.

When the index case presented to us, he had no major illness in the past. He was not taking biotin containing multivitamins or other drugs. Since he had undetectable TSH with normal free T3 and free T4, initially we considered the possibility of recovery from thyrotoxicosis or other non thyroidal illness. Patient did not have symptoms suggestive of thyrotoxicosis in the past. The tests for anti TPO and antithyroglobulin was also non suggestive. Presence of antistreptavidin antibodies, antiruthenium antibodies and heterophilic antibodies was not considered, since these conditions would also interfere with free $\mathrm{T} 3$ and free $\mathrm{T} 4$ measurement and would rather present with elevated TSH level. When patient provided the reports of TFT with undetectable TSH along normal TSH performed in different immunoassay platforms, we considered the possibility of TSH variant.

Detection of TSH using commercially available TSH immunoassays is based on a sandwich mechanism where a solid phase antibody and a detection antibody are targeted to 2 different regions of the TSH dimer. One antibody in the pair targets the beta subunit of TSH; the other antibody often targets the alpha-beta interface region of the dimer to detect intact hormone.

Mutation in TSH beta region is an identified cause of discordant result between TSH values obtained with Siemens immunoassay and other platforms.7 Drees et al. identified twenty euthyroid individuals, (19 were South Asian and one was Persian) with falsely undetectable biologically active TSH levels, who had been wrongly diagnosed with hyperthyroidism and some were started with treatment. ${ }^{7}$ After detailed investigation, it was found that mutation in TSH beta region (R55G) i.e arginine to glycine amino acid change, was responsible for discordance observed between TSH values obtained with 
Undetectable TSH could be a TSH variant; case of...

Jour of Diab and Endo Assoc of Nepal 2019; 3 (1): (34-37)

ISSN Print 2594-3367 ISSN Online 2631-2107

different immunoassay platforms. ${ }^{7}$

This arginine-55 is part of an epitope recognised by antibodies used in some immunoassays, explaining loss of TSH immunoreactivity in such platforms.

In our patient TSH determined by Siemens assay was undetectable but his serum TSH was found to be normal when determined by Roche and Abbot Immunoassay system. Thus, testing the serum with different auto analyzer should be considered when erroneous TSH result is suspected. We could not perform TSH beta region gene sequencing due to financial constraint. It was easier to counsel our patient since he was a medical student.

\section{Conclusion}

Physicians practicing endocrinology need to be aware about basic principle of immunoassay and types of interferences. In addition, collaboration between physician and laboratory personnel is needed to minimize the potential for inappropriate treatment. The high frequency of TSH-beta region mutation in our population mandates that, undetectable TSH readings in euthyroid patients should be reanalyzed using an alternate TSH assay.

\section{References}

1. Edith T, Starich GH, Mazzaferri EL. Sensitivity, specificity, and cost-effectiveness of the sensitive thyrotropin assay in the diagnosis of thyroid disease in ambulatory patients. Archives of internal medicine. 1989 Mar 1; 149(3):526-32.

2. Piketty ML, Polak M, Flechtner I, GonzalesBriceño L, Souberbielle JC. False biochemical diagnosis of hyperthyroidism in streptavidinbiotin-based immunoassays: the problem of biotin intake and related interferences. Clinical Chemistry and Laboratory Medicine (CCLM). 2017 Jun 1; 55(6):780-8.

3. Mongolu S, Armston AE, Mozley E, Nasruddin A. Heterophilic antibody interference affecting multiple hormone assays: Is it due to rheumatoid factor? Scandinavian journal of clinical and laboratory investigation. $2016 \mathrm{Apr}$
$2 ; 76(3): 240-2$.

4. Gulbahar O, Konca Degertekin C, Akturk M, Yalcin MM, Kalan I, Atikeler GF, Altinova AE, Yetkin I, Arslan M, Toruner F. A case with immunoassay interferences in the measurement of multiple hormones. The Journal of Clinical Endocrinology \& Metabolism. 2015 Jun 1; 100(6):2147-53.

5. Favresse J, Burlacu MC, Maiter D, Gruson D. Interferences with thyroid function immunoassays: clinical implications and detection algorithm. Endocrine reviews. 2018 Jul 4; 39(5):830-50.

6. Edith T, Starich GH, Mazzaferri EL. Sensitivity, specificity, and cost-effectiveness of the sensitive thyrotropin assay in the diagnosis of thyroid disease in ambulatory patients. Archives of internal medicine. 1989 Mar 1; 149(3):526-32.

7. Drees JC, Stone JA, Reamer CR, Arboleda VE, Huang K, Hrynkow J, Greene DN, Petrie MS, Hoke C, Lorey TS, Dlott RS. Falsely undetectable TSH in a cohort of South Asian euthyroid patients. The Journal of Clinical Endocrinology \& Metabolism. 2014 Apr 1; 99(4):1171-9. 